\title{
Systematic analysis of variants related to familial hypercholesterolemia in families with premature myocardial infarction
}

\author{
Ingrid Brænne ${ }^{1,2,8}$, Mariana Kleinecke ${ }^{1,2,8}$, Benedikt Reiz ${ }^{1,2}$, Elisabeth Graf ${ }^{3}$, Tim Strom ${ }^{3}$, Thomas Wieland ${ }^{3}$, \\ Marcus Fischer ${ }^{4}$, Thorsten Kessler ${ }^{5}$, Christian Hengstenberg ${ }^{5,6}$, Thomas Meitinger ${ }^{3,6,7}$, Jeanette Erdmann ${ }^{\star, 1,2,8}$ \\ and Heribert Schunkert ${ }^{5,6,8}$
}

Familial hypercholesterolemia (FH) is an oligogenic disorder characterized by markedly elevated low-density lipoprotein cholesterol (LDLC) levels. Variants in four genes have been reported to cause the classical autosomal-dominant form of the disease. FH is largely under-diagnosed in European countries. As FH increases the risk for coronary artery disease (CAD) and myocardial infarction (MI), it might be specifically overlooked in the large number of such patients. Here, we systematically examined the frequency of potential FH-causing variants by exome sequencing in $\mathbf{2 5 5}$ German patients with premature $\mathbf{M I}$ and a positive family history for CAD. We further performed co-segregation analyses in an average of 5.5 family members per MI patient. In total, we identified 11 potential disease-causing variants that co-segregate within the families, that is, $5 \%$ of patients with premature $\mathrm{MI}$ and positive CAD family history had FH. Eight variants were previously reported as disease-causing and three are novel (LDLR.c.811G >A p.(V271I)), PCSK9.c.610G >A (p.(D204N)) and STAP1.c.139A > G (p.(T47A))). Co-segregation analyses identified multiple additional family members carrying one of these FH variants and the clinical phenotype of either FH $(n=2)$ or FH and premature CAD $(n=15)$. However, exome sequencing also revealed that some variants in FH genes, which have been reported to cause $\mathrm{FH}$, do not co-segregate with $\mathrm{FH}$. The data reveal that a large proportion of $\mathrm{FH}$ patients escape the diagnosis, even when they have premature MI. Hence, systematic molecular-genetic screening for FH in such patients may reveal a substantial number of cases and thereby allow a timely LDLC-lowering in both FH/MI patients as well as their variantcarrying family members.

European Journal of Human Genetics (2016) 24, 191-197; doi:10.1038/ejhg.2015.100; published online 3 June 2015

\section{INTRODUCTION}

Familial hypercholesterolemia (FH) is an autosomal-dominant disease that leads to markedly elevated low-density lipoprotein cholesterol (LDLC) levels and increased risk for coronary artery disease (CAD) and myocardial infarction (MI). The prevalence of FH is estimated as high as one in $200-500,{ }^{1}$ with even higher frequencies in populations with founder effects. ${ }^{2} \mathrm{FH}$ is mainly caused by variants in genes coding for proteins affecting hepatic LDLC uptake including the LDL receptor (LDLR), in which most disease-causing variants are found, as well as apolipoprotein B-100 (APOB) and proprotein convertase subtilisin/ kexin type 9 (PCSK9). ${ }^{1,3-6}$ More recently, STAP1 has been proposed as a fourth gene causing $\mathrm{FH}^{7}$ Cumulatively, variants in these genes explain around $40 \%$ of $\mathrm{FH}$ cases. $^{8}$

The phenotype may vary in variant carriers or even copied by clustering of common LDL-modifying variants, each affecting LDLC levels by only a small extent. ${ }^{9}$ The large number of patients with apparently monogenic $\mathrm{FH}$ but without currently known variants also suggests that other genes, which have not been identified so far, may cause FH.

Multiple studies document the preventive effect of intensive medical LDL-lowering at young age to prevent cardiovascular events. ${ }^{10-12}$ Therefore, it has been suggested that incidental detection of variants leading to FH should be communicated to the affected individual and the family. ${ }^{13}$ In fact, owing to the high frequency of FH, several guidelines recommend programs to systematically unravel variants and to facilitate medical treatment already at young age. ${ }^{14-16}$ Despite the knowledge of causal genes and the obvious advantages of early therapy only $1-15 \%$ of $\mathrm{FH}$ cases are diagnosed in most European countries. ${ }^{17}$ Notable exceptions are Norway (43\%) and the Netherlands (71\%), in which national screening programs had been initiated. ${ }^{1,18,19}$ There are several reasons why FH is vastly under-diagnosed. First, LDLC levels and other clinical presentations of $\mathrm{FH}$ are variable. ${ }^{20}$ Second, a small family size may obscure the inherited nature of $\mathrm{FH},{ }^{21-23}$ and third, with FH being only one of multiple genetic and exogenous conditions

\footnotetext{
${ }^{1}$ Institut für Integrative und Experimentelle Genomik, Universität zu Lübeck, Lübeck, Germany; ${ }^{2}$ DZHK (German Research Centre for Cardiovascular Research), partner site Hamburg/Lübeck/Kiel, Lübeck, Germany; ${ }^{3}$ Institute of Human Genetics, Helmholtz Zentrum München, German Research Center for Environmental Health, Neuherberg, Germany; ${ }^{4}$ Klinik und Poliklinik für Innere Medizin II, Universitätsklinikum Regensburg, Regensburg, Germany; ${ }^{5}$ Deutsches Herzzentrum München, Technische Universität München, München, Germany; ${ }^{6}$ DZHK (German Centre for Cardiovascular Research), partner site Munich Heart Alliance, Munich, Germany; ${ }^{7}$ Institute of Human Genetics, Technische Universität München, Munich, Germany

8These authors contributed equally to this work.

*Correspondence: Dr J Erdmann, Institut für Integrative und Experimentelle Genomik, Universität zu Lübeck, Maria-Goeppert-Str. 1 , Lübeck 23562, Germany. Tel: +49 451 500 4857; Fax: +49 451500 5288; E-mail: jeanette.erdmann@iieg.uni-luebeck.de

Received 17 December 2014; revised 2 April 2015; accepted 17 April 2015; published online 3 June 2015
} 
affecting $\mathrm{CAD}$ risk, it might be overlooked in the large number of $\mathrm{CAD} / \mathrm{MI}$ patients.

It is assumed that $\mathrm{FH}$ explains about $20 \%$ of premature CAD cases with familial clustering. ${ }^{24,25}$ However, no systematic analysis is available to quantitate $\mathrm{FH}$ variants by molecular-genetic screening in individuals with premature CAD. In this work, we evaluate the frequency of $\mathrm{FH}$ due to variants in the $L D L R, A P O B, P C S K 9$ and STAP1 genes in 255 unselected patients with premature MI (clinical manifestation before the age of 60) and a positive family history.

\section{MATERIALS AND METHODS}

\section{Patients}

The ascertainment strategy of MI families is described elsewhere. ${ }^{26,27}$ In brief, index patients had suffered from MI before the age of 60 years. If at least one additional living sibling was affected with MI or severe CAD (defined by percutaneous coronary intervention or coronary artery bypass grafting) before the age of 70 years, the entire family (index patient, available parents and siblings) was contacted and invited to participate in the study. For the present study, we chose one affected individual each from 255 such families for wholeexome sequencing. Clinical characteristics are detailed in Table 1.

All subjects analyzed in this study gave written informed consent. The local ethical committee (University of Regensburg, Germany) approved the study.

\section{Exome sequencing}

Exome sequencing was performed as 54-bp (base pair) paired end runs on a GenomeAnalyzer IIx system (Illumina, San Diego, CA, USA) after in-solution enrichment of exonic sequences (SureSelect Human All Exon $38 \mathrm{Mb}$ kit, Agilent, Santa Clara, CA, USA), yielding on average 6.2 giga bases $(\mathrm{Gb})$ of sequence per individual. Read alignment was performed with BWA (v. 0.5.8) using the default parameters. We used the human genome assembly hg19 (GRCh37) as reference. A small percentage of duplicate reads (4-5\%) were removed. Single nucleotide variants and small insertions and deletions (indels) were detected using SAMtools (v 0.1.7). For the variant filter part of SAMtools, we used the default parameters with the exception of setting a maximum read depth to 9999. Furthermore, we required putative single nucleotide variants to fulfil the following criteria: (i) median base quality of the variant bases of at least 15; (ii) a minimum of $15 \%$ of reads showing the variant base; and (iii) the variant base is indicated by at least $5 \%$ of reads coming from different strands.

\section{Variant validation}

We used annovar ${ }^{28}$ to annotate the single nucleotide variant. Annotation was based on several databases provided by annovar such as UCSC known gene, ${ }^{29}$ dbSNP (http://www.ncbi.nlm.nih.gov/SNP/), Exome Sequencing Project (http://evs.gs.washington.edu/EVS/) and 1000 Genomes. ${ }^{30}$ In addition, we also

\section{Table 1 Clinical characteristics of the MI patients analyzed in this} work
Clinical characteristics

Age at inclusion

Sex

Male, $n(\%)$

Female, $n(\%)$

$\mathrm{BMI}$

Age at first $\mathrm{MI}$

$\operatorname{LDLC}^{\mathrm{b}}$ (mg/dl)

Statin therapy, $n$ (\%)

LDLC $>190$

Smoking, $n(\%)$
Diabetes, $n(\%)$
MI patients $\left(n=255^{a}\right)$

53.6

78.3

21.7

27.5

42.5

175.8

62.9

42.5

9.9

82.6

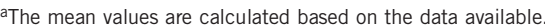

bAdjusted for statin intake according to the CURVES study. ${ }^{35}$ annotated several functional prediction scores such as SIFT, ${ }^{31} \mathrm{CADD},{ }^{32}$ PolyPhen $2^{33}$ and Mutation Taster. ${ }^{34}$ Variant validation was performed using PCR and Sanger sequencing. For co-segregation analysis, validated variants were screened in affected and unaffected family members.

Primers that were used for validation are listed in Supplementary Table 1. PCR was carried out in $20 \mu \mathrm{l}$ volume containing $50 \mathrm{ng}$ genomic DNA, $1 \mu \mathrm{l}$ of each primer and either $8 \mu \mathrm{l}$ of Mastermix (5PRIME, Hamburg, Germany) or $0.1 \mu \mathrm{l}$ Taq-Polymerase and $4 \mu \mathrm{l}$ Taq-buffer mix (Bioline Pharmaceutical AG, Baar, Switzerland). Samples were processed in a Sensoquest labcycler with a standard touchdown PCR program (annealing temperature from 59 to $65^{\circ} \mathrm{C}$ ).

The variants identified in this work were submitted to the publicly funded database LOVD (http://databases.lovd.nl/shared/variants/) with the LOVD individual IDs 00033731, 00033768, 00033771-00033807.

\section{RESULTS AND DISCUSSION}

We studied 255 unselected MI/CAD patients from families with strong familial clustering of MI/CAD. The average age at disease manifestation was 42.5 years. The families of the index patients had an average size of 5.5 individuals with an average number of 2.3 affected family members.

Whole-exome sequencing yielded on average $6.2 \mathrm{~Gb}$ of sequence per individual. The average read depth was 78 with between 84.5 and $85.6 \%$ of the target regions covered at least $20 \times$. In total, we identified 259 single nucleotide variants in the LDLR, APOB, PCSK9 and STAP1 genes.

We considered individuals with LDLC levels of $>160 \mathrm{mg} / \mathrm{dl}$ after adjusting for statin intake for further analysis. ${ }^{35}$ To identify potential disease-causing variants, we filtered these variants based on three assumptions. First, we expect a strong functional impact and therefore, we removed synonymous and intronic variants outside splice-site regions. Second, we expect the variant to be rare in the general population, as FH affects 1 in 200 individuals at the most. Hence, we filtered based on a frequency of $1 \%$ in public databases. Third, we filtered variants in segmental duplications due to the high falsepositive rate in these regions.

Additionally, a decision tree (Supplementary Figure 1) was developed to further filter the remaining 54 variants. This decision tree includes several considerations, such as the predicted deleterious/ damaging effect of a variant on the protein function or whether the variant is known as potentially disease-causing in HGMD. ${ }^{36}$ Figure 1 shows the pedigrees of families where a potential disease-causing variant was identified.

\section{Variant spectrum in LDLR, APOB, PCSK9 and STAP1 genes}

In total, we identified 13 rare variants with potentially functional effect in the $L D L R, 9$ variants in $A P O B, 8$ variants in PCSK9 and 1 variant in STAP1. Twenty-three variants are missense variants, three are deletions, two are nonsense variants, two are splice-site variants and one is an insertion. Of these 31 variants, 24 were confirmed using Sanger sequencing (see Table 2). Fourteen have been previously reported to cause FH and listed in HGMD (access date March 2014).

\section{$L D L R$ gene variants}

Co-segregating new variant: c.811G $>A$ (p.(V271I)). We identified only one variant in the LDLR gene that has not been previously reported (c.811G $>$ A (p.(V271I))). Both CAD/MI-affected members of family 7421 available for the genetic study carry this variant. Both individuals show elevated LDLC levels of 286 and $261 \mathrm{mg} / \mathrm{dl}$ after adjustment for statin treatment. The variant is also found in an family member unaffected by CAD but with markedly elevated LDLC levels $(220 \mathrm{mg} / \mathrm{dl})$.

Variant c.811G $>$ A (p.(V271I)) lies in the domain that interacts with $\mathrm{APOB}^{37}$ and is in close proximity with several disease-causing amino acid changes listed in HGMD, for instance p.N272T, p.C270S, 

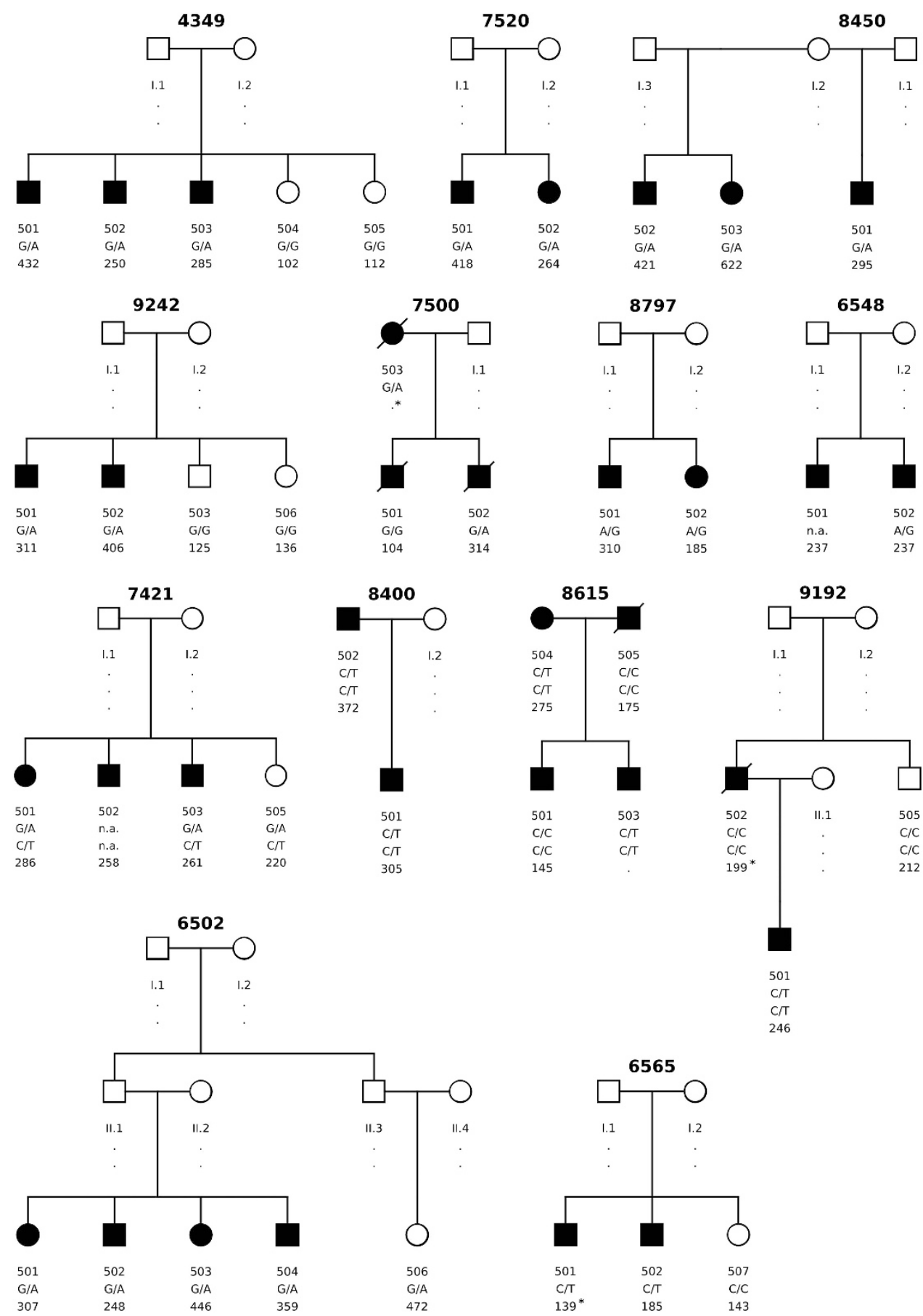

Figure 1 Pedigrees of families where a potential disease-causing variant was identified. Squares are males and circles are females. Individuals affected with MI/CAD are shown as dark symbols.. The first row of numbers below the symbols are the individual IDs, the second line show the genotype for the identified variant and the third row the corrected LDLC level. (LDLC levels marked with * are not corrected for statin treatment.) Detailed information is shown in Table 2. The potential disease-causing variant per family are: Family ID 4349: LDLR. c.131G>A (p.(W44*)); Family ID7520: LDLR. c.1285G>A (p.(V429M)); Family ID8450: LDLR. c.G1775 (p.(G592E)); Family ID 9242: LDLR. c.1444G>A (p.(D482N)); Family ID 7500: PCSK9. c.610G >A (p.(D204N)); Family ID 8797: STAP1. c.139A>G (p.(T47A)); Family ID 6548 LDLR. c.2231G>A (p.(R744Q)); Family ID 7421: LDLR. c.811G>A (p.(V271I)); Family IDs 8400, 8615, 9192: APOB. c.10580G >A (p.(R3527Q))/(APOB. c.7696G >A (p.(E2566K))); Family ID6502: LDLR.c.1359-1G >A; Family ID 6565: LDLR. c.757C > T (p.(R253W)).

p.C270R, p.C276R, p.G269D or p.C270Y. ${ }^{38-43}$ On the basis of the position of the identified variant with respect to previously reported variants, it is very likely that c.811G $>\mathrm{A}(\mathrm{p} .(\mathrm{V} 271 \mathrm{I}))$ is responsible for the increase of LDLC in the variant carriers.
Co-segregating known variants: c.1285G $>A$ (p.(V429M)), c.1444G $>A$

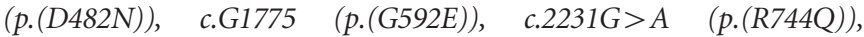
$\begin{array}{lllll}c .757 C>T & (p .(R 253 W)), & c .131 G>A & \left(p .\left(W 44^{*}\right)\right), & c .798 T>A\end{array}$ $(p .(D 266 E))$ and c.828C $>A \quad\left(p .\left(C 276^{*}\right)\right)$. The missense variants 


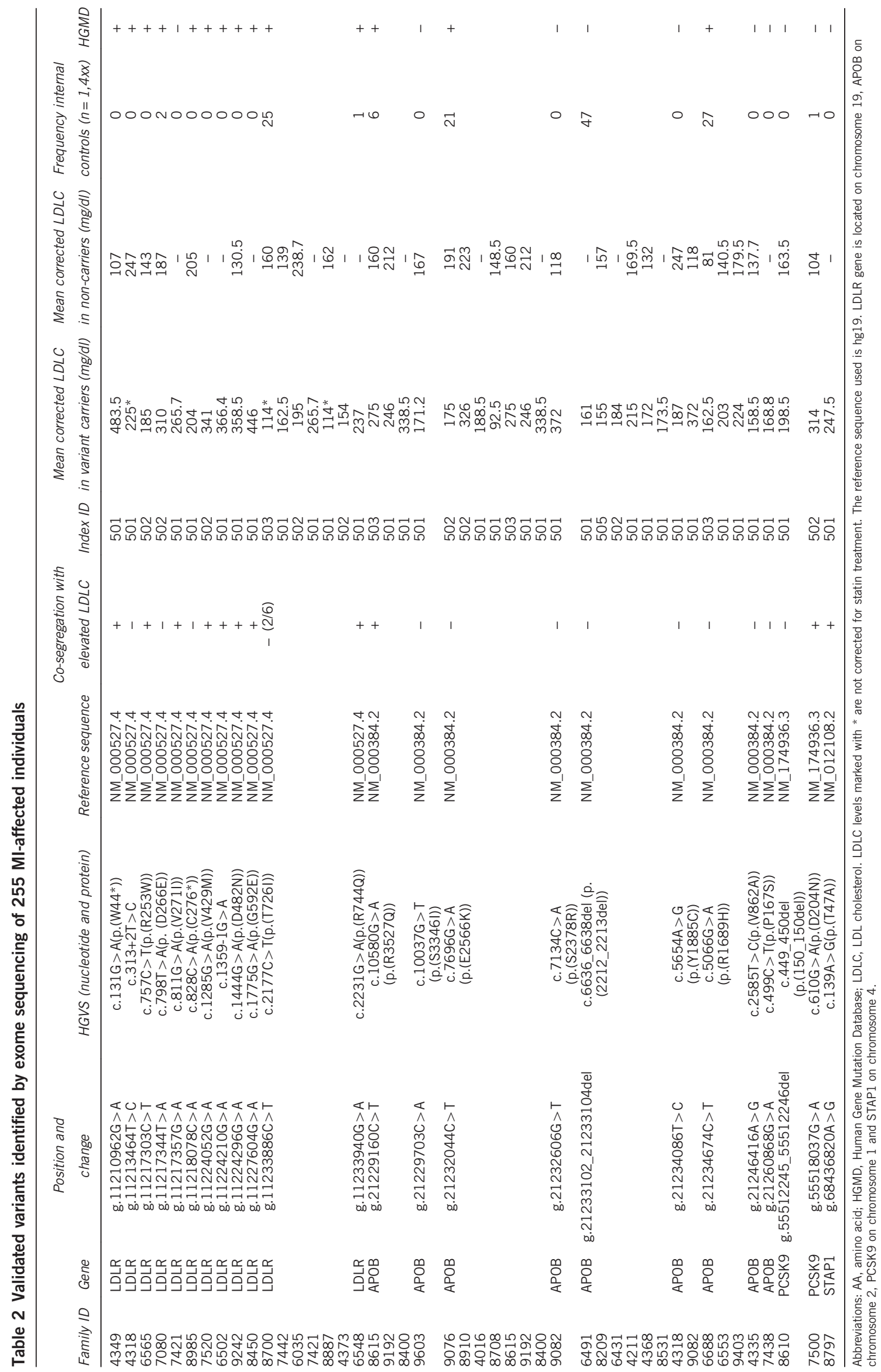


c.1285G $>$ A (p.(V429M)) (first found in the African population ${ }^{44}$ ), c.1444G $>$ A (p.(D482N)), c.G1775 (p.(G592E)), c.2231G $>$ A (p.(R744Q)), c.757C > T (p.(R253W)) and c.131G >A (p. (W44*)) show co-segregation with elevated LDLC levels (mean $344 \mathrm{mg} / \mathrm{dl}$ after adjustment for statin treatment) and MI in the families under study. Hence, on the basis of our own results and evidence in previously reported studies, ${ }^{38,44-46}$ these variants probably cause the disease in these patients/families.

The splice site variant c.1359-1G >A has also been reported to cause $\mathrm{FH} .{ }^{47}$ In addition, we have previously also identified this variant as probably disease-causing in one of our extended MI/FH families. ${ }^{48}$ Co-segregation analysis showed that all five family members carry the variant. Four family members are affected with MI/CAD and all have elevated LDLC levels even after statin therapy (mean LDLC of $366 \mathrm{mg} / \mathrm{dl}$ ). Hence, our data confirm previous findings.

The missense variant (c.798T $>\mathrm{A}(\mathrm{p} .(\mathrm{D} 266 \mathrm{E})))$ and the nonsense variant $\left(\mathrm{c} .828 \mathrm{C}>\mathrm{A}\left(\mathrm{p} .\left(\mathrm{C} 276^{*}\right)\right)\right)$ were also found in individuals with markedly elevated LDLC levels and MI. Both variants have been reported as disease-causing in HGMD. ${ }^{38,49}$ Here, we only identified both variants in the index patients of family 7080 and 8985 and not in the two other affected family members. Both index patients have markedly elevated LDLC levels of 310 and $204 \mathrm{mg} / \mathrm{dl}$, respectively. Interestingly, the two non-carriers within these families also have elevated LDLC levels of 187 and $205 \mathrm{mg} / \mathrm{dl}$.

Whereas c. $828 \mathrm{C}>\mathrm{A}\left(\mathrm{p} .\left(\mathrm{C} 276^{*}\right)\right)$ is not found in our internal non $\mathrm{CAD} / \mathrm{FH}$ patients, c.798T $>\mathrm{A}$ (p.(D266E)) is found twice. However, as $\mathrm{FH}$ is a common disease, we also expect to find a small number of $\mathrm{FH}$ cases in population-based controls.

Non-co-segregating known variant: rs45508991. One LDL receptor variant, c. $2177 \mathrm{C}>\mathrm{T}$ (p.(T726I),rs45508991), is found in 6 of the 255 patients. This variant is reported to cause $\mathrm{FH}$ and is labelled as likely disease-causing in HGMD but the variant may be only pathogenic in combination with another variant in the LDLR gene. ${ }^{50,51}$ In our data, we checked for co-segregation with LDLC and observed a positive co-segregation only in one family (family 7421) that also carries an additional co-segregating LDLR variant (c.811G > A (p.(V271I))). The other five families show poor or no evidence that the variant causes FH. In fact, in carriers of this variant, LDLC levels range from 147 to $268 \mathrm{mg} / \mathrm{dl}$, and in non-carriers from 86 to $257 \mathrm{mg} / \mathrm{dl}$.

Despite incomplete co-segregation with elevated LDLC levels, c. $2177 \mathrm{C}>\mathrm{T}$ (p.(T726I)) is only found in one MI/CAD-unaffected family member (Supplementary Figure 2). In our data set, we do not find variant carriers with neither MI/CAD nor FH. To further check whether c.2177C > T (p.(T726I)) is associated with an increased risk of $\mathrm{CAD}$ or $\mathrm{FH}$, we compared the number of variant carriers in our sample within the general population. As c.2177C $>\mathrm{T}$ (p.(T726I)) is mainly found in individuals of European ancestry, ${ }^{50}$ we compared the frequency only in European samples. With a frequency of 0.007 in 1000 Genomes $(1 \mathrm{kG})$ data set (European samples, phase 3 version 5) versus $0.023(6 / 255)$ in our sample set, there is evidence that the variant indeed increases the risk of $\mathrm{CAD} / \mathrm{FH}$. However, we also found c. $2177 \mathrm{C}>\mathrm{T}$ (p.(T726I)) in 25 of $1462(0.017)$ internal European nonCAD patients $(P$-value $=0.477)$. Hence, as the variant is equally common in affected versus unaffected individuals, we expect the accumulation of c.2177C $>\mathrm{T}$ ( $\mathrm{p}$.(T726I)) rather to be a founder effect than related to an increased risk of $\mathrm{CAD} / \mathrm{FH}$.

Non-co-segregating known variant: c.313+2T >C. LDLR variant c.313 $+2 \mathrm{~T}>\mathrm{C}$ does neither co-segregate with elevated LDLC levels nor with $\mathrm{MI} / \mathrm{CAD}$. This splice-site variant is only found in the index patient and not in the two relatives with elevated LDLC levels (mean LDLC $217 \mathrm{mg} / \mathrm{dl}$ ) of which one is also MI/CAD-affected. Variant c.313 $+2 \mathrm{~T}>\mathrm{C}$ is reported to cause $\mathrm{FH}^{52}$ and is labelled as disease-causing in HGMD. However, on the basis of the results of co-segregation analysis in family 4318, we do not expect the variant to be disease-causing, at least not to be the primary cause of MI/CAD in this family.

Double variant: LDLR. c.131G>A (p. $\left.\left(W 44^{*}\right)\right)$ and PCSK9.c.137G $>$ T (p. $(R 46 L))$. For all validated LDLR-variant carriers, we checked for a second variant in PCSK9, APOB and STAP1 gene. We found one compound heterozygote patient with a variant in the $L D L R$ gene (c.131G >A (p. (W44*))) and PCSK9 gene (c.137G>T (p.(R46L))) (family 4349, patient 501). The PCSK9 variant, c.137G>T (p.(R46L)), is reported to be associated with a significant reduction of LDLC. ${ }^{53}$ Co-segregation analysis revealed that one of the three c.131G $>$ A (p. $\left.\left(\mathrm{W} 44^{*}\right)\right)$ carriers also carry $c .137 G>T(p .(R 46 L))$ (patient 501), in addition to one family member without the LDLR variant (patient 504).

Reduced PCSK9 activity leads to increased density of LDLR ${ }^{54,55}$ at the cell membrane. As we have heterozygous variant carriers expressing one healthy LDLR allele, we would not expect to see a strong effect, but expect to see reduced levels of LDLC for the double variant carriers. However, the LDLC level is markedly elevated for all three c.131G > A (p. (W44*)) variant carriers (mean LDLC $322 \mathrm{mg} / \mathrm{dl}$ ). We do not identify a higher level of LDLC in the affected relative with the protective PCSK9 variant (LDLC level of $432 \mathrm{mg} / \mathrm{dl}$ ). On the contrary, this patient has the highest LDLC level in the family. This could, however, imply that the protective effect of the PCSK9 variant is negligible compared with the LDLC level increase caused by the LDLR variant. The unaffected relative carrying only the reported protective variant has similar LDLC levels as the second unaffected relative lacking both variants. In summary, we do not see a reduction in LDLC levels in $c .137 G>T$ (p. $(R 46 L))$ carriers and would not expect the variant to markedly reduce LDLC levels.

\section{APOB gene variants}

Of the nine APOB variants, three were found in HGMD: c.7696G > A (p. (E2566K)) and c.5066G $>$ A (p. (R1689H)) are reported to be associated with high triglyceride and c.10580G > A (p.(R3527Q)) with high LDLC levels. Of the nine variants, only one co-segregates with $\mathrm{FH}$ and MI/LDLC levels, and is previously reported. In the following, we will only discuss the three HGMD variants.

c.10580G $>A$ (p.(R3527Q)) and c.7696G >A (p.(E2566K)). Interestingly, c.10580G $>$ A (p.(R3527Q)) is always found in combination with the c.7696G > A (p. (E2566K)) variant. Vice versa, c.7696G > A (p. (E2566K)) is also found without the c.10580G $>$ A (p.(R3527Q)) variant. As c.10580G >A (p.(R3527Q)) appears to be on the same allele as c.7696G > A (p.(E2566K)), c.10580G >A (p.(R3527Q)) seems to be the more recent variant.

The c.10580G > A (p.(R3527Q)) variant was found in three families (8615, 9192, 8400). Variant c.10580G $>$ A (p.(R3527Q)) has been reported to cause defective binding of ApoB to the LDLR. ${ }^{56}$ All five c.10580G < A (p.(R3527Q)) carriers show elevated LDLC levels (mean LDLC $300 \mathrm{mg} / \mathrm{dl}$ ). However, two siblings with elevated LDLC (mean $206 \mathrm{mg} / \mathrm{dl}$ ), do not carry the variant. These non-carriers could, however, have another cause of disease. Hence, there is evidence that c.10580G > A (p.(R3527Q)) may cause elevated LDLC levels. Therefore, our results support previous findings.

Variant c.7696G > A (p.(E2566K)) was identified in seven families. This variant does neither co-segregate with elevated LDLC levels nor 
with MI. In the seven families, we find three affected individuals (mean $214 \mathrm{mg} / \mathrm{dl}$ ) without the variant as well as four unaffected variant carriers (mean $133 \mathrm{mg} / \mathrm{dl}$ ).

Non-co-segregating known variant: c.5066G $>A(p .(R 1689 H))$. The c.5066G >A (p. (R1689H)) variant was found in three of the 255 exome-sequenced MI-patients. Two non-carriers have LDLC levels above $190 \mathrm{mg} / \mathrm{dl}$ while four variant carriers do not show elevated cholesterol levels (mean $156 \mathrm{mg} / \mathrm{dl}$ ). Hence, we do not expect this variant to be the cause of disease in these families nor increase the risk of $\mathrm{FH} / \mathrm{MI}$ in general.

\section{PCSK9 gene variant}

We identified two variants in the PCSK9 gene. Both variants have not been reported earlier. On the basis of co-segregation analysis with LDLC levels, the c.610G > A (p. (D204N)) variant might cause FH. The affected variant carrier has LDLC levels of $314 \mathrm{mg} / \mathrm{dl}$ and the MIaffected brother has low LDLC levels ( $104 \mathrm{mg} / \mathrm{dl})$. Hence, the cause of MI may differ in this individual. The variant c.449_450del (p.(150_150del) does not co-segregate with FH in the family.

\section{STAP1 gene variant}

Variant c.139A $>\mathrm{G}$ (p.(T47A)) is the only variant we found in the STAP1 gene and has not been reported previously. Both family members available for genetic studies are MI-affected and carry the amino acid substitution. In addition, both show elevated LDLC levels (mean $248 \mathrm{mg} / \mathrm{dl}$ ). Hence, we might have identified the causal variant in this family but functional studies are necessary to further evaluate its functional implication.

\section{Potential polygenic cause of $\mathrm{FH}$}

Of the analyzed CAD patients, $48 \%$ have LDLC levels above $190 \mathrm{mg} / \mathrm{dl}$ after correction for statin intake ${ }^{35}$ (LDLC levels were available for 212 $\mathrm{CAD}$ patients). We find rare potential disease-causing variants in $12.7 \%$ of these patients CAD patients with high LDLC levels. It has recently been shown that the clinical phenotype of $\mathrm{FH}$ can also be caused by the accumulation of common variants with small effects. ${ }^{9}$ Indeed, it has been reported that a score of six SNPs enables to discriminate $\mathrm{FH}$ patients from healthy controls. ${ }^{57}$ We calculated the score as described by Futema et al for the patients with high LDLC levels but without a rare disease-causing variant and compared the score with the controls of the German MI Family Study II ${ }^{58}$ $(n=1298)$. Not all SNPs were covered by exome sequencing, so we calculated the score based on GeneChip Human Mapping $500 \mathrm{~K}$ Array Set (Affymetrix) available for 234 of the $255 \mathrm{CAD}$ patients. The mean score in controls $(0.63)$ are significantly lower than the mean score $(0.69)$ in patients with high LDLC levels $(P$-value $=0.025)$. Our values are in range with the scores reported by Futema et al ( 0.63 in the control cohort and 0.71 in the mutation negative $\mathrm{FH}$ patients). Hence, the mutation-negative patients with elevated LDLC levels might have a polygenic cause of disease.

\section{CONCLUSION}

Here, we screened 255 patients with premature MI/CAD for variants in genes known to cause FH. If we only account for variants in the LDLR gene, $3.1 \%$ of the patients carry potential $\mathrm{FH}$-causing variants. If we also account for variants in APOB, PCSK9 and STAP1, we have a cumulative frequency of $\mathrm{FH}$-causing variants of $5.1 \%$. Indeed, the frequency of potential disease-causing variants in our sample is probably underestimated. Large rearrangements are reported to account for $11 \%$ of the LDLR variants. ${ }^{59}$ Unfortunately, the coverage profile of our data was not uniform enough to allow a screening for such variants and hence we might have missed some of these.

In summary, the high frequency of potential $\mathrm{FH}$-causing variants in these unselected MI/CAD patients supports the hypothesis that $\mathrm{FH}$ is overseen in a substantial number of patients with MI/CAD and demonstrates that genetic screening also of MI/CAD patients can improve diagnosis of $\mathrm{FH}$.

Additionally, we also screened family members of the index patients for the identified variants. This revealed that 17 family members also carry the potential FH-causing variant. Hence, our findings underline the need for a systematic molecular-genetic screening to enable an early diagnosis of $\mathrm{FH}$ and to allow timely preventive treatment.

A further interesting finding is that the functional effect of several variants reported as disease-causing, for instance in HGMD, is questionable. We observed that five reported causal variants show none or minor functional impact in our analyzed families. Consequently, given the far-reaching implications of the diagnosis of $\mathrm{FH}$, each variant has to be carefully evaluated. In fact, a co-segregation analysis is advisable to determine whether a variant truly is diseasecausing.

In summary, our work demonstrates that exome sequencing can be used for FH-variant screening. In addition, the quality of the exome sequencing has improved over the last years, allowing identification not only of small nucleotide variants but also large rearrangements. ${ }^{60}$ Also, as the sequencing costs have decreased dramatically, exome sequencing might become the method of choice for molecular genetic screening of, for instance, $\mathrm{FH}$.

\section{CONFLICT OF INTEREST}

The authors declare no conflict of interest.

\section{ACKNOWLEDGEMENTS}

We thank all the family members who participated in this research. Without the continuous support of these patients over more than 15 years, the present work would not have been possible. We like to thank Sandra Wrobel for technical assistance. We also would like to thank Drs Björn Mayer, Ute Hubauer and Anika Großhennig for help with the German Myocardial Infarction Study. The study is supported by the German Federal Ministry of Education and Research (BMBF) in the context of the e:Med program (e:AtheroSysMed and sysINFLAME) and the FP7 European Union project CVgenes@target (261123). Further grants were received by the local focus program 'Medizinische Genetik' of the Universität zu Lübeck, as well as by the Fondation Leducq (CADgenomics: Understanding Coronary Artery Disease Genes, 12CVD02). This study was also supported through the Deutsche Forschungsgemeinschaft (DFG) cluster of excellence 'Inflammation at Interfaces'.

\section{AUTHOR CONTRIBUTIONS}

JE, IB and HS designed the study; IB, MK and BR performed the bioinformatic analysis; EG, TS, TM and TW performed the NGS; MK, MF and CH collected the family data; IB, MK, JE and HS wrote the manuscript. All authors read and approved the final manuscript.

\footnotetext{
1 Nordestgaard BG, Chapman MJ, Humphries SE et al: Familial hypercholesterolaemia is underdiagnosed and undertreated in the general population: guidance for clinicians to prevent coronary heart disease: consensus statement of the European Atherosclerosis Society. Eur Heart J 2013; 34: 3478-3490a.

2 Foody JM: Familial hypercholesterolemia: an under-recognized but significant concern in cardiology practice. Clin Cardiol 2014; 37: 119-125.

3 De Castro-Oros I, Pocovi M, Civeira F: The genetic basis of familial hypercholesterolemia: inheritance, linkage, and mutations. App Clin Genet 2010; 3: 53-64.
} 
4 Austin MA, Hutter CM, Zimmern RL, Humphries SE: Genetic causes of monogenic heterozygous familial hypercholesterolemia: a HuGE prevalence review. Am J Epidemio 2004; 160: 407-420.

5 Umans-Eckenhausen MA, Defesche JC, Sijbrands EJ, Scheerder RL, Kastelein JJ Review of first 5 years of screening for familial hypercholesterolaemia in the Netherlands. Lancet 2001; 357: 165-168.

6 Leren TP, Manshaus T, Skovholt $U$ et al: Application of molecular genetics for diagnosing familial hypercholesterolemia in Norway: results from a family-based screening program. Semin Vasc Med 2004; 4: 75-85.

7 Fouchier SW, Dallinga-Thie GM, Meijers JC et al: Mutations in STAP1 Are Associated With Autosomal Dominant Hypercholesterolemia. Circ Res 2014; 115: 552-555.

8 Santos RD, Maranhao RC: What is new in familial hypercholesterolemia? Curr Opin Lipidol 2014; 25: 183-188.

9 Talmud PJ, Shah S, Whittall R et al: Use of low-density lipoprotein cholesterol gene score to distinguish patients with polygenic and monogenic familial hypercholesterolaemia: a case-control study. Lancet 2013; 381: 1293-1301.

10 Versmissen J, Oosterveer DM, Yazdanpanah $\mathrm{M}$ et al: Efficacy of statins in familial hypercholesterolaemia: a long term cohort study. BMJ 2008; 337: a2423.

11 Wiegman A, de Groot E, Hutten BA et al: Arterial intima-media thickness in children heterozygous for familial hypercholesterolaemia. Lancet 2004; 363: 369-370.

12 Marks D, Thorogood M, Neil HA, Humphries SE: A review on the diagnosis, natural history, and treatment of familial hypercholesterolaemia. Atherosclerosis 2003; 168 $1-14$

13 Green RC, Berg JS, Grody WW et al: ACMG recommendations for reporting of incidental findings in clinical exome and genome sequencing. Genet Med 2013; 15 565-574.

14 Reiner Z, Catapano AL, De Backer G et al: [ESC/EAS Guidelines for the management of dyslipidaemias]. Revista espanola de cardiologia 2011; 64: 1168.e1-1168.e60.

15 Minhas R, Humphries SE, Qureshi N, Neil HA, Group NGD: Controversies in familial hypercholesterolaemia: recommendations of the NICE Guideline Development Group for the identification and management of familial hypercholesterolaemia. Heart 2009; 95: 584-587, discussion 587-591.

16 National-Institute-of-Health. Third Report of the Expert Panel on Detection, Evaluation, and Treatment of High Blood Cholesterol in Adults (Adult Treatment Panel III) 2004.

17 Brice P, Burton H, Edwards CW, Humphries SE, Aitman TJ: Familial hypercholesterolaemia: a pressing issue for European health care. Atherosclerosis 2013; 231: 223-226.

18 Goldberg AC, Hopkins PN, Toth PP et al: Familial hypercholesterolemia: screening, diagnosis and management of pediatric and adult patients: clinical guidance from the National Lipid Association Expert Panel on Familial Hypercholesterolemia. J Clin Lipidol 2011; 5: S1-S8.

19 Benn M, Watts GF, Tybjaerg-Hansen A, Nordestgaard BG: Familial hypercholesterolemia in the danish general population: prevalence, coronary artery disease, and cholesterol-lowering medication. J Clin Endocrinol Metab 2012; 97: 3956-3964.

20 Nauck MS, Koster W, Dorfer $\mathrm{K}$ et al: Identification of recurrent and novel mutations in the LDL receptor gene in German patients with familial hypercholesterolemia. Hum Mutat 2001; 18: 165-166.

21 Pötzsch O, Weinmann J, Haustein T: Geburtentrends und familiensituation in deutschland. Statistisches Bundesamt, Wiesbaden 2013, 2013.

22 Office-for-National-Statistics. Family size in 2012, 2013

23 Testa MR: Family sizes in Europe: evidence from the 2011 Eurobarometer Survey. European Demographic Research Papers 2. Vienna: Vienna Inst of Demography, 2012

24 Goldstein JL, Schrott HG, Hazzard WR, Bierman EL, Motulsky AG: Hyperlipidemia in coronary heart disease. II. Genetic analysis of lipid levels in 176 families and delineation of a new inherited disorder, combined hyperlipidemia. J Clin Invest 1973; 52: 1544-1568.

25 Neefjes LA, Ten Kate GJ, Alexia R et al: Accelerated subclinical coronary atherosclerosis in patients with familial hypercholesterolemia. Atherosclerosis 2011; 219: 721-727.

26 Broeckel U, Hengstenberg C, Mayer B et al: A comprehensive linkage analysis for myocardial infarction and its related risk factors. Nat Genet 2002; 30: 210-214.

27 Fischer M, Broeckel U, Holmer S et al: Distinct heritable patterns of angiographic coronary artery disease in families with myocardial infarction. Circulation 2005; 111 : 855-862.

28 Wang K, Li M, Hakonarson H: ANNOVAR: functional annotation of genetic variants from high-throughput sequencing data. Nucleic Acids Res 2010; 38: e164.

29 Karolchik D, Barber GP, Casper J et al: The UCSC Genome Browser database: 2014 update. Nucleic Acids Res 2014; 42: D764-D770.

30 Abecasis GR, Altshuler D, Auton A et al: 1000 Genomes Project Consortium A map of human genome variation from population-scale sequencing. Nature 2010; 467 1061-1073.

$31 \mathrm{Ng} \mathrm{PC}$, Henikoff S: Predicting deleterious amino acid substitutions. Genome Res 2001; 11: 863-874.

32 Kircher M, Witten DM, Jain P, O'Roak BJ, Cooper GM, Shendure J: A general framework for estimating the relative pathogenicity of human genetic variants. Nat Genet 2014; 46: $310-315$.

33 Adzhubei IA, Schmidt S, Peshkin L et al: A method and server for predicting damaging missense mutations. Nat Methods 2010; 7: 248-249.
34 Schwarz JM, Rodelsperger C, Schuelke M, Seelow D: MutationTaster evaluates diseasecausing potential of sequence alterations. Nat Methods 2010; 7: 575-576.

35 Pincus J: Comparative dose efficacy study of atorvastatin versus simvastatin, pravastatin, lovastatin, and fluvastatin in patients with hypercholesterolemia (the CURVES study). Am J Cardiol 1998; 82: 406-407.

36 Loux N, Saint-Jore B, Collod G et al: Screening for new mutations in the LDL receptor gene in seven French familial hypercholesterolemia families by the single strand conformation polymorphism method. Hum Mutat 1992; 1: 325-332.

37 Russell DW, Brown MS, Goldstein JL: Different combinations of cysteine-rich repeats mediate binding of low density lipoprotein receptor to two different proteins. J Biol Chem 1989; 264: 21682-21688.

38 Hobbs HH, Brown MS, Goldstein JL: Molecular genetics of the LDL receptor gene in familial hypercholesterolemia. Hum Mutat 1992; 1: 445-466.

39 Duskova L, Kopeckova L, Jansova E et al: An APEX-based genotyping microarray for the screening of 168 mutations associated with familial hypercholesterolemia. Atherosclerosis 2011; 216: 139-145.

40 Fouchier SW, Kastelein JJ, Defesche JC: Update of the molecular basis of familial hypercholesterolemia in The Netherlands. Hum Mutat 2005; 26: 550-556.

41 Kuhrova V, Francova H, Zapletalova $\mathrm{P}$ et al: Spectrum of low density lipoprotein receptor mutations in Czech hypercholesterolemic patients. Hum Mutat 2001; 18 : 253.

42 Bertolini S, Cantafora A, Averna M et al: Clinical expression of familial hypercholesterolemia in clusters of mutations of the LDL receptor gene that cause a receptor-defective or receptor-negative phenotype. Arterioscler Thromb Vasc Biol 2000; 20: E41-E52.

43 Mozas P, Castillo S, Tejedor D et al: Molecular characterization of familial hypercholesterolemia in Spain: identification of 39 novel and 77 recurrent mutations in LDLR. Hum Mutat 2004; 24: 187.

44 Leitersdorf E, Van der Westhuyzen DR, Coetzee GA, Hobbs HH: Two common low density lipoprotein receptor gene mutations cause familial hypercholesterolemia in Afrikaners. J Clin Invest 1989; 84: 954-961.

45 Ward AJ, O'Kane M, Young I, Nicholls DP, Nevin NC, Graham CA: Three novel mutations in the EGF precursor homology domain of the low-density lipoprotein receptor gene in Northern Irish patients with familial hypercholesterolemia. Hum Mutat 1995; 6: 254-256.

46 Sun XM, Patel DD, Knight BL, Soutar AK: Influence of genotype at the low density lipoprotein $(L D L)$ receptor gene locus on the clinical phenotype and response to lipidlowering drug therapy in heterozygous familial hypercholesterolaemia. The Familial Hypercholesterolaemia Regression Study Group. Atherosclerosis 1998; 136: 175-185.

47 Peeters AV, Van Gaal LF, du Plessis L, Lombardi MP, Havekes LM, Kotze MJ: Mutational and genetic origin of LDL receptor gene mutations detected in both Belgian and Dutch familial hypercholesterolemics. Hum Genet 1997; 100: 266-270.

48 Brænne I, Reiz B, Medack A et al: Whole-exome sequencing in an extended family with myocardial infarction unmasks familial hypercholesterolemia. BMC Cardiovasc Disord 2014; 14: 108.

49 Mozas P, Cenarro A, Civeira F, Castillo S, Ros E, Pocovi M: Mutation analysis in 36 unrelated Spanish subjects with familial hypercholesterolemia: identification of 3 novel mutations in the LDL receptor gene. Hum Mutat 2000; 15: 483-484.

50 Tejedor MT, Cenarro A, Tejedor D et al: Haplotype analyses, mechanism and evolution of common double mutants in the human LDL receptor gene. Mol Genet Genomics 2010; 283: 565-574.

51 Alharbi KK, Aldahmesh MA, Spanakis $\mathrm{E}$ et al: Mutation scanning by meltMADGE: validations using BRCA1 and LDLR, and demonstration of the potential to identify severe, moderate, silent, rare, and paucimorphic mutations in the general population. Genome Res 2005; 15: 967-977.

52 Lombardi P, Sijbrands EJ, van de Giessen $\mathrm{K}$ et al: Mutations in the low density lipoprotein receptor gene of familial hypercholesterolemic patients detected by denaturing gradient gel electrophoresis and direct sequencing. J Lipid Res 1995; 36: 860-867.

53 Cohen JC, Boerwinkle E, Mosley THJr., Hobbs HH: Sequence variations in PCSK9, low LDL, and protection against coronary heart disease. N Engl J Med 2006; 354: $1264-1272$

54 Youngblom E, Knowles JW: Familial hypercholesterolemia In: Pagon RA, Adam MP, Bird TD (eds): GeneReviews. Seattle (WA): University of Washington. 1993.

55 Wu NQ, Li JJ: PCSK9 gene mutations and low-density lipoprotein cholesterol. Clin Chim Acta 2014; 431C: 148-153.

56 Soria LF, Ludwig EH, Clarke HR, Vega GL, Grundy SM, McCarthy BJ: Association between a specific apolipoprotein $B$ mutation and familial defective apolipoprotein B-100. Proc Natl Acad Sci USA 1989; 86: 587-591.

57 Futema M, Shah S, Cooper JA et al: Refinement of variant selection for the LDL cholesterol genetic risk score in the diagnosis of the polygenic form of clinical familial hypercholesterolemia and replication in samples from 6 countries. Clin Chem 2015; 61: 231-238.

58 Erdmann J, Großhennig A, Braund PS et al: New susceptibility locus for coronary artery disease on chromosome 3q22.3. Nat Genet 2009; 41: 280-282.

59 Goldmann R, Tichy L, Freiberger T et al: Genomic characterization of large rearrangements of the LDLR gene in Czech patients with familial hypercholesterolemia. BMC Med Genet 2010; 11: 115

60 Futema M, Plagnol V, Whittall RA et al: Use of targeted exome sequencing as a diagnostic tool for Familial Hypercholesterolaemia. J Med Genet 2012; 49: 644-649.

Supplementary Information accompanies this paper on European Journal of Human Genetics website (http://www.nature.com/ejhg) 